\title{
Una primera experiencia de innovación docente en Construcción
}

\section{A first experience of teaching innovation in Construction}

Pedro Gómez de Terreros Guardiola

ORCID: https://orcid.org/0000-0002-6275-8779

E.T.S. Arquitectura

Departamento Construcciones Arquitectónicas I

Universidad de Sevilla

pgomez@us.es

Fecha de recepción: 18/11/2010

Fecha de aceptación: 21/11/2019

DOI: http://dx.doi.org/10.12795/9788447221912.092

Pp.: 2076-2095 


\section{Resumen}

Tras 25 años impartiendo clase en la Escuela de Arquitectura y 20 en la asignatura de Construcción 1, he detectado que los alumnos ingresados desde 2010 (nacidos sobre 1992) tenían mayor dificultad para asimilar mis explicaciones (Carr, 2011). Por ello, durante los últimos años, he ido introduciendo pequeños cambios en la forma de dar clase para mejorar los resultados, no siempre con éxito y, lógicamente, manteniendo sólo aquellas innovaciones que funcionaban. Preocupado por los progresivos cambios que sigo detectando en los alumnos de primer curso, careciendo de formación en técnicas docentes y animado por mis compañeros que ya lo habían realizado, me inscribí en el CGDU para entender mejor a los alumnos, aprender qué y por qué algunas técnicas funcionan y aplicarlas correctamente en mi actividad docente. En este trabajo se presenta el ciclo de mejora diseñado y desarrollado así como los resultados de su aplicación.

Palabras clave: Construcción 1, Grado de Arquitectura, Docencia Universitaria, Experimentación Docente Universitaria.

\section{Abstract}

After 25 years teaching in the School of Architecture and 20 in the subject of Construction 1, I found that the students signed up since 2010 (born about 1992) had trouble in assimilating my explanations (Carr, 2011). Therefore, over the last few years, in order to improve the results, I have been introducing small changes in the way of teaching, not always successfully, and obviously maintaining only those innovations that worked. Concerned about the progressive changes that I continue detecting in first year students, lacking training in teaching techniques, and encouraged by my peers who have already done so, I enrolled in the CGDU to better understand the students behaviour, to learn which and why techniques work, and to apply them correctly in my teaching activity. This work presents the cycle of improvement designed and developed as well as the results of its application.

Key words: Construction I, Architecture Degree, University Teaching; University Teaching Experimentation

Jornadas de Formación e Innovación Docente del Profesorado | № 2 (2019) Esta obra se distribuye con la licencia Creative Commons Reconocimiento-NoComercial-SinObraDerivada 4.0 Internacional (CC BY-NC-ND 4.0.) 


\section{Descripción del contexto}

La asignatura en la que se ha realizado el ciclo de mejora es Construcción 1, que corresponde al primer cuatrimestre de primer curso. Los alumnos están distribuidos en 12 grupos de 25-30 estudiantes. Tiene 6 eurocréditos teórico-prácticos, repartidos en 15 semanas con docencia en sesiones de dos horas, en dos días durante 15 semanas. Es una asignatura troncal que comienza el primer día de clase y se desarrolla durante todo el cuatrimestre.

El grupo que vamos a analizar tiene asignada un aula con dimensiones muy ajustadas, los 28 alumnos comparten por parejas unos tableros que les permiten dibujar. El espacio tiene el equipamiento básico: proyector, pizarra y habría que mejorar bastante su aislamiento acústico con al aula adyacente.

Recordemos siempre que los alumnos con los que vamos a tratar acaban de ingresar y que esta es su primera experiencia universitaria. Para la gran mayoría también es la primera vez que se enfrentan al hecho constructivo y carecen de conocimientos básicos tanto del objeto de la asignatura (vocabulario, técnicas, normativa, materiales..) como de conocimientos instrumentales básicos: dibujo, fisica, informática, etc. A cambio, la mayoría del contenido es novedoso, relativamente sencillo y de inmediata aplicación. La asignatura suele tener muy buena aceptación por los alumnos.

\section{Diseño previo del Ciclo de Mejora Docente (CMD)}

Siguiendo el calendario y las instrucciones del curso, he tenido que elegir un tema desarrollado en 8 horas que es relativamente sencillo: Muros de fábricas pesadas revestidas, que, para los "profanos", lo podríamos traducir como "paredes realizadas en la obra mediante la unión de 
piezas más pequeñas". Para su aprendizaje se plantea un necesario contenido teórico y una práctica consistente en aplicar esta técnica constructiva a una vivienda modelo.

Dentro de la programación docente, es en este tema cuando los alumnos se encuentran por primera vez con un elemento constructivo, su diseño y su materialidad. Se pretende que vean la complejidad de los elementos reales más allá de su mera forma apreciando las múltiples facetas y funciones que contienen y la complejidad que puede surgir de una pieza tan simple como un ladrillo. Más concretamente deben ver cómo le influyen los materiales, la forma y formato de las piezas, la normativa, los encuentros, las juntas, su relación con otros elementos (puertas, ventanas, instalaciones...). En definitiva, las distintas facetas de la construcción y las enormes posibilidades que ésta ofrece.

\section{Mapa de contenidos y modelo metodológico}

Nunca antes había realizado un mapa de contenidos, tampoco los había estudiado y, salvo por algunas miradas fugaces, desconocía casi todo acerca de ellos. Evitando la tentación de plagiar alguno similar, intenté esquematizar TODOS los contenidos de la sesión y elaboré este primer diseño (ver Figura 1).

Evidentemente, esta primera relación pormenorizada resultaba confusa, es poco intuitiva e implica tantos datos que la hace dificilmente asimilable por los alumnos. Es por ello que he elaborado un segundo mapa de contenidos que no pretende ser tan exhaustivo, apareciendo ahora sólo los conceptos generales y sus relaciones e incorporando la secuencia de actividades de forma que podemos ver en qué momento y secuencia tratamos los diversos contenidos que pretendemos que adquieran (ver Figura 2). 
Para el diseño del modelo metodológico (De Alba y Porlán, 2017) ya tenía alguna experiencia previa, y el adoptado (ver Figura 3) intenta reproducir el ideal que se planteó tras un primer Ciclo de Mejora en el Aula (CIMA). Comienza con una introducción inicial tutorizada y debatida con los alumnos, para pasar a la presentación de un problema práctico a resolver conjuntamente con los alumnos en las dos sesiones intermedias, retomando las explicaciones teóricas e interpretativas de forma que se abran nuevas posibilidades y aspectos distintos a los desarrollados en las actividades por los alumnos.

Jornadas de Formación e Innovación Docente del Profesorado | № 2 (2019) Esta obra se distribuye con la licencia Creative Commons 


\section{Pedro Gómez de TeRreros Guardiola}
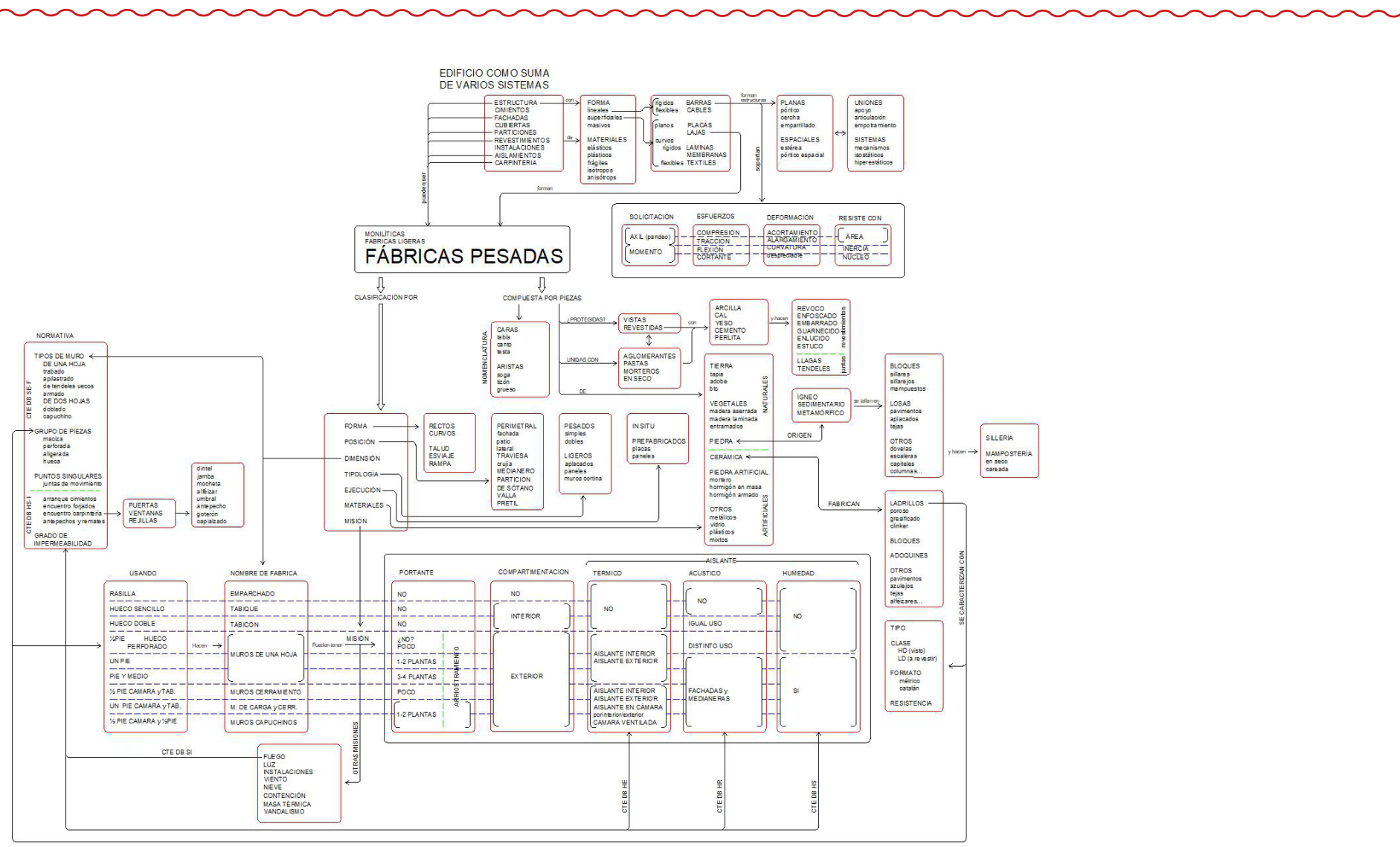

Figura 1. Mapa de contenidos inicial

Jornadas de Formación e Innovación Docente del Profesorado I № 2 (2019)

(C) $(1)$

cc) (i) $\Theta$ Reconocimiento-NoComercial-SinObraDerivada

Internacional (CC BY-NC-ND 4.0.) 
actividad 4.1

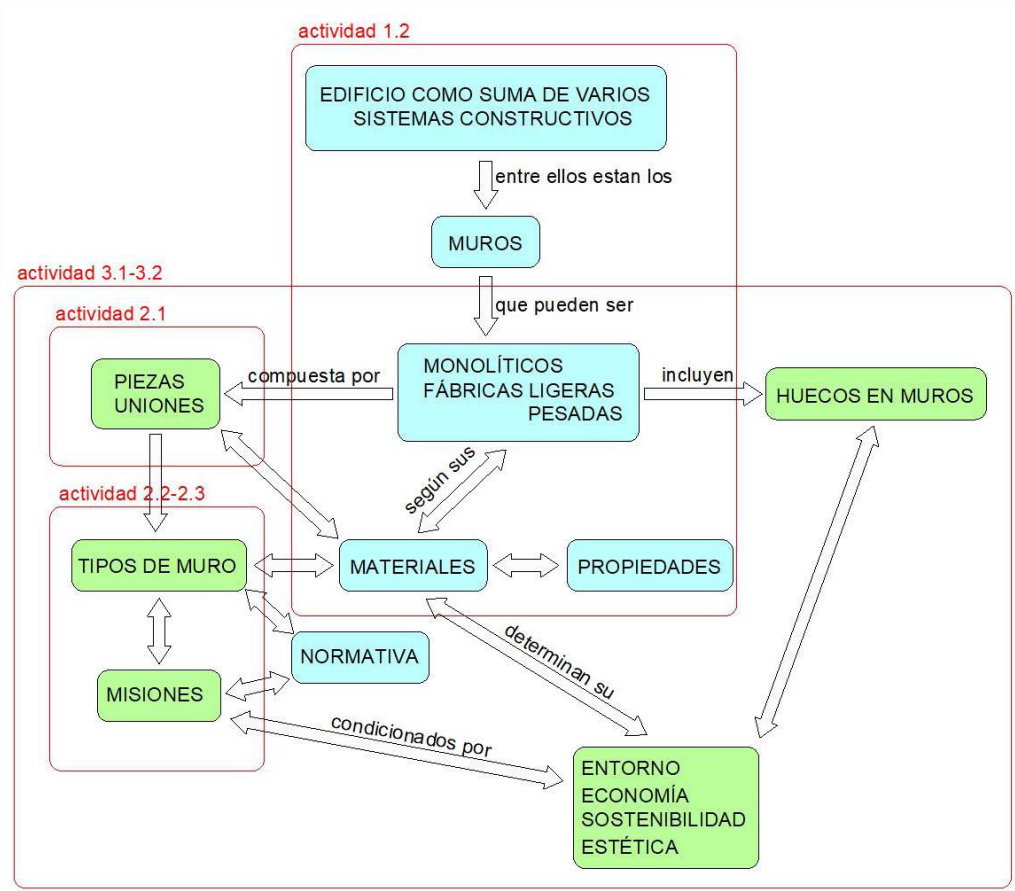

Figura 2. Mapa general de contenidos

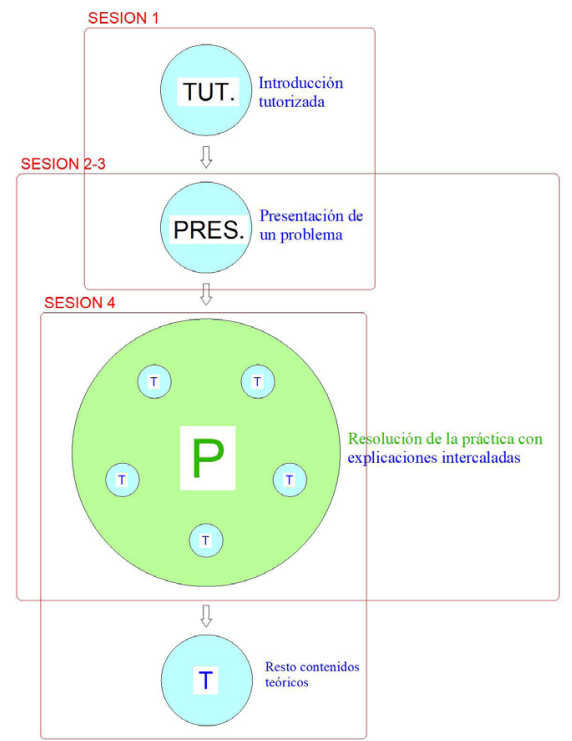

Figura 3. Modelo metodológico

Jornadas de Formación e Innovación Docente del Profesorado | № 2 (2019) Esta obra se distribuye con la licencia Creative Commons 
Secuencia de actividades programadas y cuestionario

La secuencia de actividades diseñada se recoge en la Tabla 1.

\section{Tabla 1. Secuencia de actividades}

\begin{tabular}{|c|c|c|}
\hline 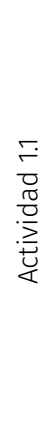 & $\begin{array}{l}\text { Introducción al edificio } \\
\text { actual en el que los distintos } \\
\text { elementos constructivos han } \\
\text { ido especializándose, dando } \\
\text { lugar a varios sistemas que, } \\
\text { aunque se estudian de forma } \\
\text { independiente, en la realidad } \\
\text { siempre están entrelazados. }\end{array}$ & $\begin{array}{l}\text { Empezamos con una pregunta clave (Bain, } \\
\text { 2007) ¿Para qué construimos? ¿Cuáles } \\
\text { son las funciones del edificio? Lo ideal } \\
\text { sería haber usado los resultados del } \\
\text { cuestionario para poner en común las } \\
\text { respuestas, pero a la hora de comenzar } \\
\text { el ciclo suponía erróneamente que no } \\
\text { podíamos usar las respuestas para no } \\
\text { alterar la evaluación del aprendizaje y } \\
\text { pasarlo nuevamente de forma "sorpresa" } \\
\text { al final de la última sesión }\end{array}$ \\
\hline 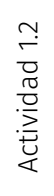 & $\begin{array}{l}\text { Debate sobre el sistema } \\
\text { estructural, tipos, elementos, } \\
\text { funcionamiento. }\end{array}$ & $\begin{array}{l}\text { Explicación de los principios de los } \\
\text { sistemas estructurales de barras (son el } \\
\text { modelo más sencillo) y comparación en } \\
\text { introducción a los sistemas estructurales } \\
\text { murales. Ventajas e inconvenientes. }\end{array}$ \\
\hline
\end{tabular}

SEGUNDA SESIÓN (2h)

\begin{tabular}{|c|c|c|}
\hline 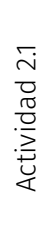 & $\begin{array}{l}\text { Visualización de diversas } \\
\text { posibilidades dentro de una } \\
\text { misma familia. }\end{array}$ & $\begin{array}{l}\text { Se aportarán diversos tipos de piezas } \\
\text { de cerámica reales para que los } \\
\text { alumnos sugieran las características } \\
\text { más importantes que las caracterizan. } \\
\text { Evidentemente este proceso es dirigido y } \\
\text { estimulado por el profesor. }\end{array}$ \\
\hline 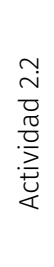 & $\begin{array}{l}\text { Exposición de los productos } \\
\text { comerciales más habituales } \\
\text { para practicar. }\end{array}$ & $\begin{array}{l}\text { Aplicación de la explicación anterior para } \\
\text { que los estudiantes propongan formas } \\
\text { de agrupación lógicas asociándolas a sus } \\
\text { funciones. } \\
\text { El profesor resumirá los resultados } \\
\text { obtenidos completando los contenidos } \\
\text { esenciales. }\end{array}$ \\
\hline
\end{tabular}

Jornadas de Formación e Innovación Docente del Profesorado | № 2 (2019) Esta obra se distribuye con la licencia Creative Commons Reconocimiento-NoComercial-SinObraDerivada Internacional (CC BY-NC-ND 4.0.) 


\begin{tabular}{|c|c|c|}
\hline 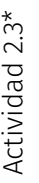 & $\begin{array}{l}\text { Caracterizar las piezas } \\
\text { con las que se construye } \\
\text { el modelo de curso y las } \\
\text { paredes que se elaboran a } \\
\text { partir de éstas. }\end{array}$ & $\begin{array}{l}\text { Dibujar a escala las piezas y los muros } \\
\text { con los que se construye el modelo } \\
\text { indicando capas, materiales, espesores y } \\
\text { especificaciones. }\end{array}$ \\
\hline
\end{tabular}

\section{TERCERA SESIÓN (2h)}

\begin{tabular}{|c|c|c|}
\hline 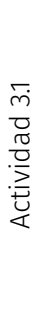 & $\begin{array}{l}\text { Aplicar los resultados a la } \\
\text { realizad constructiva viendo } \\
\text { las dificultades y cambios } \\
\text { que surgen en el modelo. }\end{array}$ & $\begin{array}{l}\text { Redibujar el modelo de curso con las } \\
\text { medidas y características de los muros } \\
\text { elaborados en su casa, marcando cámaras } \\
\text { de aire, mochetas, paso de instalaciones, } \\
\text { uniones, etc. Esta tarea se hace más } \\
\text { compleja y requiere la ayuda -a veces } \\
\text { general y otras veces individualizada- del } \\
\text { profesor. }\end{array}$ \\
\hline 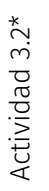 & $\begin{array}{l}\text { Terminar las actividades que } \\
\text { queden pendientes de las } \\
\text { sesiones } 2 \text { y } 3 \text {. }\end{array}$ & $\begin{array}{l}\text { En clase solamente se desarrolla la } \\
\text { planta baja del modelo. En casa deben } \\
\text { enfrentarse solos a la planta alta que } \\
\text { tiene los mismos elementos pero en otra } \\
\text { situación. }\end{array}$ \\
\hline
\end{tabular}

\section{CUARTA SESIÓN (2h)}

\begin{tabular}{|c|c|c|}
\hline 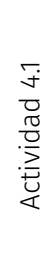 & $\begin{array}{l}\text { Teoría. Explicación de } \\
\text { los diferentes tipos de } \\
\text { muro, sus materiales, } \\
\text { las diferentes formas, } \\
\text { clasificaciones, normativa, } \\
\text { etc. haciendo hincapié en las } \\
\text { interrelaciones entre ellos. }\end{array}$ & $\begin{array}{l}\text { Puesta en común de las respuestas a la } \\
\text { pregunta } 1 \text { del cuestionario: ¿Para qué se } \\
\text { construye una pared? Introducimos de } \\
\text { nuevo el debate para introducir y facilitar } \\
\text { la comprensión e importancia de las } \\
\text { relaciones entre las múltiples facetas que } \\
\text { se desarrollarán en el desarrollo teórico. }\end{array}$ \\
\hline
\end{tabular}

De esta forma, las tres columnas ordenan las actividades, explican los contenidos que se incluyen para cada una de ellas, y el proceso que se pretende seguir para su desarrollo.

Para hacer el seguimiento de la evolución de los estudiantes y tener una idea de sus planteamientos iniciales, se elaboró un cuestionario con seis preguntas sencillas (muchas obvias) abiertas. Las preguntas y las valoraciones planteadas se recogen en la Tabla 2. 
Tabla 2. Cuestionario inicial y final

\begin{tabular}{|c|c|c|c|}
\hline & Pregunta & Respuestas & Objetivo \\
\hline 1 & $\begin{array}{l}\text { ¿Para qué se } \\
\text { construye una } \\
\text { pared? }\end{array}$ & $\begin{array}{l}\text { a) No responde o responde } \\
\text { algo sin sentido } \\
\text { b) Responde algo lógico } \\
\text { c) Responde una misión } \\
\text { d) Responde dos o más } \\
\text { misiones } \\
\text { e) Responde a las tres mi- } \\
\text { siones reales }\end{array}$ & $\begin{array}{l}\text { Con esta pregunta se } \\
\text { trata de que el alumno } \\
\text { se enfrente por primera } \\
\text { vez a la complejidad del } \\
\text { elemento construido y } \\
\text { que reflexione sobre las } \\
\text { múltiples facetas que se } \\
\text { pueden desarrollar de este } \\
\text { elemento tan simple }\end{array}$ \\
\hline 2 & $\begin{array}{l}\text { ¿Con qué } \\
\text { materiales } \\
\text { puedes } \\
\text { construirla? }\end{array}$ & $\begin{array}{l}\text { a) No responde } \\
\text { b) Un material } \\
\text { c) Dos materiales } \\
\text { d) Tres o más materiales } \\
\text { e) Incluye materiales mixtos }\end{array}$ & $\begin{array}{l}\text { Las cosas se construyen } \\
\text { con "algo". Hay muchas } \\
\text { y diversas posibilidades. } \\
\text { Abrir el abanico de éstas } \\
\text { antes de centrarse en } \\
\text { las "habituales" que - } \\
\text { lógicamente- serán las que } \\
\text { la mayoría desarrolle. }\end{array}$ \\
\hline 3 & $\begin{array}{l}\text { Dibuja e } \\
\text { identifica una } \\
\text { pieza con la } \\
\text { que podrías } \\
\text { construirla }\end{array}$ & $\begin{array}{l}\text { a) No responde } \\
\text { b) Sólo dibuja } \\
\text { c) Dibuja el tipo (huecos) } \\
\text { d) Además da otra ca- } \\
\text { racterística (medidas, } \\
\text { dimensiones...) } \\
\text { e) Lo especifica } \\
\text { correctamente }\end{array}$ & $\begin{array}{l}\text { Es un ejercicio de } \\
\text { materializar las ideas } \\
\text { anteriores dándole una } \\
\text { forma. Con ello creamos } \\
\text { una pieza modelo que } \\
\text { tiene características reales: } \\
\text { dimensiones, peso, etc. }\end{array}$ \\
\hline 4 & $\begin{array}{l}\text { Con esa pieza, } \\
\text { ¿qué tipo } \\
\text { de paredes } \\
\text { puedes } \\
\text { construir? }\end{array}$ & $\begin{array}{l}\text { a) No responde } \\
\text { b) Sólo un tipo } \\
\text { c) Varios tipos por grosores } \\
\text { o funciones } \\
\text { d) Varios tipos por grosores } \\
\text { y funciones } \\
\text { e) Nombra varios tipos con } \\
\text { grosores y funciones }\end{array}$ & $\begin{array}{l}\text { Se trata de Se trata de } \\
\text { entender que, partiendo } \\
\text { de un solo elemento y } \\
\text { mediante un proceso } \\
\text { similar al que ocurre en la } \\
\text { obra, con procedimientos } \\
\text { relativamente sencillos } \\
\text { de agrupación, seriación, } \\
\text { modulación, etc., es posible } \\
\text { obtener muy diversas } \\
\text { construcciones. }\end{array}$ \\
\hline
\end{tabular}

Jornadas de Formación e Innovación Docente del Profesorado I № 2 (2019) Esta obra se distribuye con la licencia Creative Commons Reconocimiento-NoComercial-SinObraDerivada Internacional (CC BY-NC-ND 4.0.) 


\begin{tabular}{|c|c|c|c|}
\hline 5 & $\begin{array}{l}\text { Dibuja una } \\
\text { sección vertical } \\
\text { de una de } \\
\text { esas paredes } \\
\text { identificando } \\
\text { sus } \\
\text { componentes }\end{array}$ & $\begin{array}{l}\text { a) No responde } \\
\text { b) Dibuja solo dos líneas } \\
\text { c) Dibuja las piezas, juntas y } \\
\text { revestimientos } \\
\text { d) Acota y/o nombra correc- } \\
\text { tamente los materiales } \\
\text { e) Acota y nombra correcta- } \\
\text { mente los materiales }\end{array}$ & $\begin{array}{l}\text { Al igual que en la tercera } \\
\text { pregunta, se pretende } \\
\text { materializar la idea. }\end{array}$ \\
\hline 6 & $\begin{array}{l}\text { ¿Para qué sirve } \\
\text { esa pared? }\end{array}$ & $\begin{array}{l}\text { a) No responde } \\
\text { b) Responde algo lógico } \\
\text { c) Responde sólo una mi- } \\
\text { sión correcta } \\
\text { d) Responde dos o más mi- } \\
\text { siones correctas } \\
\text { e) Responde a las tres mi- } \\
\text { siones correctamente }\end{array}$ & $\begin{array}{l}\text { Es una pregunta de } \\
\text { retroalimentación (Bain, } \\
\text { 2007), sirve para comprobar } \\
\text { el proceso y volver a } \\
\text { plantearse la primera } \\
\text { cuestión. }\end{array}$ \\
\hline
\end{tabular}

\section{Aplicación del CMD}

\section{Desarrollo de las sesiones}

Lo acontecido en las cuatro sesiones fue distinto de lo planteado. Comenzaron con el cuestionario, que se desarrolló con normalidad, los alumnos no se extrañaron y lo realizaron tranquilamente y no precisaron muchas aclaraciones. Tras éste, se habló por primera vez de los sistemas constructivos y ante las preguntas clave se originaron multitud de respuestas muy dispersas que abarcaban el ámbito arquitectónico.

Como el debate y el cuestionario se prolongaron bastante más de lo previsto, tuve que pasar directamente a la explicación sobre los sistemas estructurales. Pese a recortar los contenidos al máximo e intentar acelerar la explicación, no dio tiempo a enunciarlos todos y la aceleración de su exposición hicieron que cundiera un cierto desánimo general. Ciertamente el debate es interesante, pero limita mucho la exposición de contenidos. 
Una posible solución sería que hubiesen leído (¿reflexionado?) el tema con anterioridad (Finkel, 2008) pero tenemos el inconveniente de que ya están muy cargados de actividades. Pese a ello, creo que es posible hacerlo puntualmente, y éste es uno de los temas que lo requieren.

Para la segunda sesión, llevé varios tipos de ladrillos, lo que hizo la clase más amena y participativa pero -a cambio- el debate volvió a ocupar más tiempo del previsto. En este caso lo pude prever y, pese a la pérdida de los contenidos, fue posible impartir (dibujando en la pizarra y progresivamente) la materia prevista.

El ambiente fue mejor que en la sesión anterior y, a medida que las paredes se hacían más complejas, los alumnos iban identificando por sí solos capas y revestimientos. Aparentemente comprendieron lo que se les enseñaba y la tarea que tenían que desarrollar por sí mismos.

En la tercera sesión tuve un profesor de apoyo (becario que ya impartió la asignatura el año pasado con otro profesor). Para esta clase, la mayoría de los alumnos habían traído resuelta (aunque no acabada) la práctica que se les encomendó. La velocidad a la hora de dibujar fue muy desigual, por lo que tuvimos dificultades debido a la dispersión de los progresos. En esto ayudó que fuésemos dos profesores atendiendo individualmente a los alumnos; a cambio, la brecha entre los adelantados y los más lentos fue ampliándose.

El ambiente en clase fue muy participativo y los alumnos estuvieron las dos horas trabajando sobre las prácticas. Para los profesores, y también para algunos alumnos, fue algo estresante por el continuo ir de un alumno a otro, aunque satisfactoria.

En la cuarta y a última sesión se desarrolló el contenido teórico de las dos sesiones anteriores. Ante la experiencia 
de las clases anteriores y las encuestas finales que había que realizar al final de la clase, el debate fue más breve y la clase del tipo magistral con proyección en vez de pizarra. Quizás por ello, y por las "prisas" para poder impartir el contenido básico que no se impartió en las sesiones anteriores, la clase fue más tediosa y los alumnos estuvieron menos implicados, salvo en la parte en la que hicimos una pequeña aplicación de normativa.

La sensación es que hay una "velocidad límite" en la exposición a partir de la cual se pierde la atención. En esto, la pizarra es un instrumento muy potente pues ralentiza y ordena la explicación. De alguna forma, la docencia "lenta" es más satisfactoria para ambas partes pero implica recortar contenidos o hacer que los estudiantes los asimilen por sí solos, y esto último es complejo cuando están (o se consideran) sobrecargados de actividades.

Al final de la clase pasamos la segunda encuesta. Se notaba que ya estaban cansados y deseando acabar (era última hora de la mañana) lo que es posible que haya influido en el resultado de la misma. En esta sesión entregaron la primera tarea y el resultado ha sido muy positivo. La segunda tarea (la entregaron más tarde) también tuvo un resultado bueno, aunque no tanto como la primera, posiblemente debido a su mayor complejidad.

\section{Evaluación del aprendizaje}

La evaluación se ha hecho con el mismo cuestionario (sin que ellos lo esperasen) al final de la cuarta sesión y comparando los resultados con las respuestas iniciales. Los resultados de las encuestas iniciales (en rojo) y finales (en verde) son representados en las siguientes escaleras de aprendizaje: 

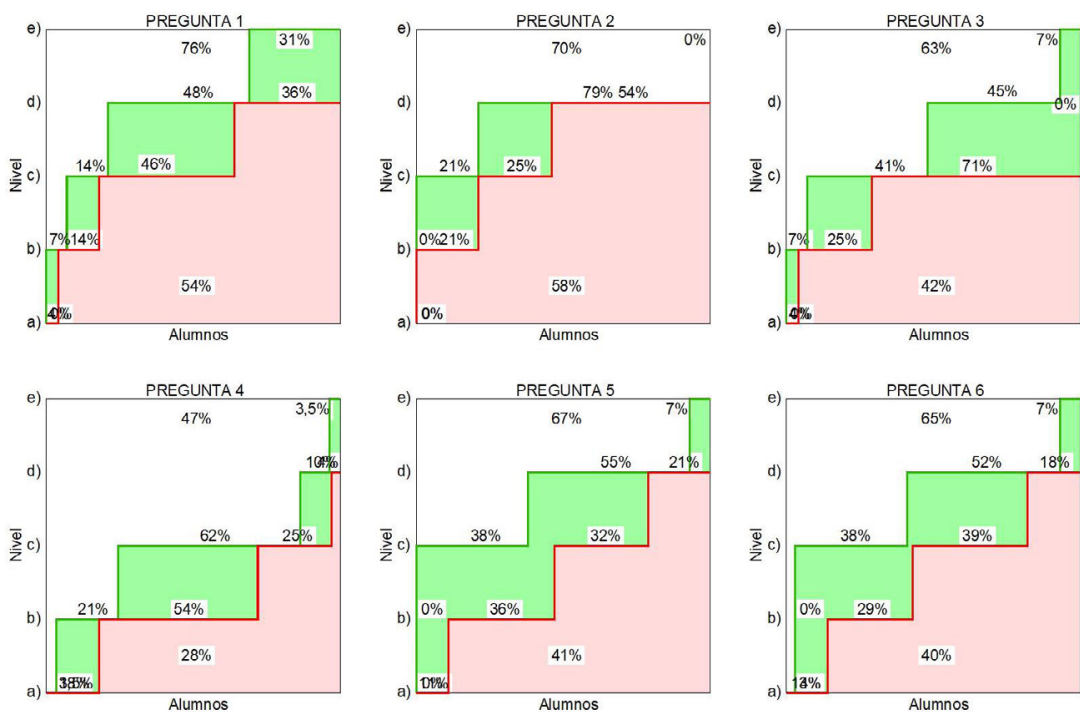

Figura 4. Escaleras de aprendizaje

En este diseño de las escaleras, el eje vertical representa la complejidad de la respuesta (en principio todas igual) y la horizontal en porcentaje de alumnos que han superado este nivel. De esta forma, si medimos el área coloreada bajo la escalera, obtenemos el porcentaje de conocimiento previo y final que tiene el conjunto de la clase sobre el total de los evaluables. Así es posible medir el estado de conocimiento inicial, y usarlo para evitar reiteraciones y establecer un punto de partida bastante preciso. Igualmente podemos ver dónde se produce un mayor o menor avance, estableciendo los conocimientos que les dificultan el progreso y ayudándolos en la labor de aprendizaje (Rivero y Porlán, 2017). Hay una característica bastante frecuente que se repite con frecuencia en las seis preguntas de este cuestionario; la hemos detectado al ver que en la representación de las escaleras la posición de las tabicas (las líneas verticales que forman los peldaños) está muy próxima en las escaleras previas y finales. Esto indica que aquellos alumnos que tras nuestra enseñanza han subido un escalón, se atascan con frecuencia en el 
siguiente. También podemos usarlas para detectar las dificultades de aprendizaje. Tomemos como ejemplo un desarrollo comentado de la escalera obtenida con la pregunta 1.

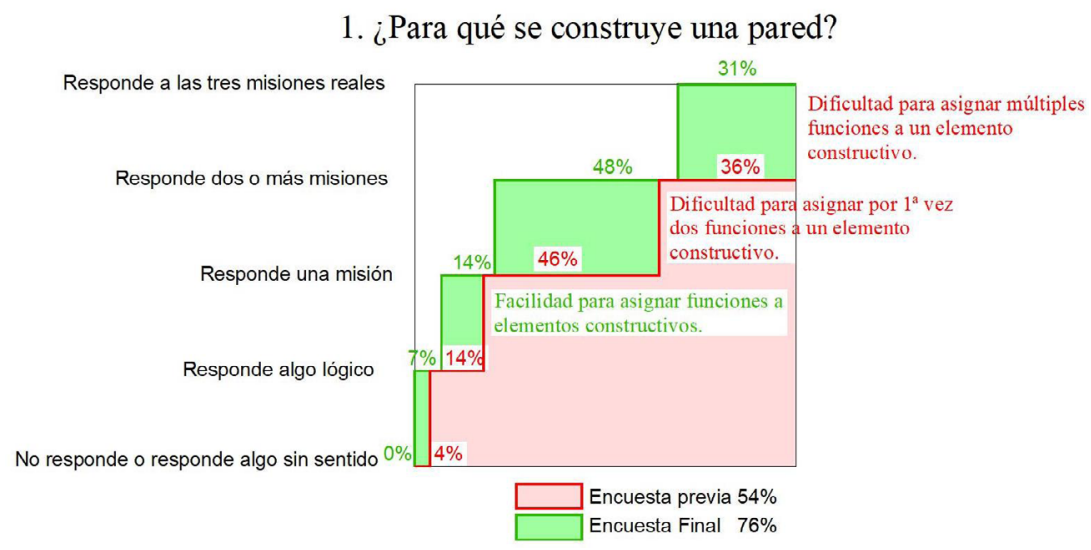

Figura 5. Escalera de la pregunta 1 comentada

Hasta ahora hemos visto que en la encuesta inicial se refleja que los alumnos saben que una pared tiene alguna función y la gran mayoría de ellos la identifica correctamente. Sin embargo les cuesta pensar que las paredes sirven para algo más, y muy pocos (en este caso ninguno) ha pensado que tuviesen más de dos funciones distintas.

Con la escalera final, veremos que el primer obstáculo ha sido superado por la mayoría de la clase y que un número considerable ha llegado a alcanzar los objetivos de aprendizaje. De hecho podríamos medirlo y así sabríamos que los alumnos ya conocían por si solos un $54 \%$ de los conceptos que eran nuestros objetivos y que tras nuestro ciclo de mejora han alcanzado un $76 \%$ de los que pretendíamos. En otras palabras, hemos conseguido que los aumenten en un $22 \%$.

También podemos tantear los progresos general o individualizado usando una tabla en la que se recojan los peldaños que sube cada alumno o en otra que recoger 
los avances medios (ver Tabla 3). En ella, cuando un estudiante ha subido un peldaño de la escalera, lo indicamos con +1. Análogamente, si sube 2 ó 3, indicamos +2 ó +3. Cuando hemos introducido -1 , es porque el estudiante ha "bajado" un peldaño. Cuando la celda aparece en blanco es porque el estudiante no ha experimentado ningún cambio en esa pregunta.

Tabla 3. Progreso individualizado

\begin{tabular}{|c|c|c|c|c|c|c|}
\hline Alumno & \multicolumn{6}{|c|}{ Progresión del alumno en cada pregunta } \\
\hline & 1 & 2 & 3 & 4 & 5 & 6 \\
\hline 1 & & & & +1 & +1 & +3 \\
\hline 2 & +2 & +1 & & & +2 & +1 \\
\hline 3 & & & & +2 & +2 & \\
\hline 4 & & +1 & & +1 & +2 & +1 \\
\hline 5 & +1 & +1 & & +2 & & +1 \\
\hline 6 & -1 & & +1 & & +1 & +1 \\
\hline 7 & +1 & & +3 & +2 & +2 & +1 \\
\hline 8 & & +1 & +2 & +1 & +2 & +1 \\
\hline 9 & +1 & +1 & & +1 & +2 & +1 \\
\hline 10 & +1 & & & & +2 & +1 \\
\hline 11 & +2 & & +1 & +1 & +2 & \\
\hline 12 & +2 & +2 & & & & \\
\hline 13 & +1 & & +2 & +1 & +2 & \\
\hline 14 & & +1 & +1 & +1 & +1 & +1 \\
\hline 15 & & & & & & \\
\hline 16 & +1 & & +1 & +1 & & \\
\hline 17 & +1 & & +2 & & & +1 \\
\hline 18 & & & & +2 & +2 & +1 \\
\hline 19 & +1 & +1 & +1 & +1 & +1 & -1 \\
\hline
\end{tabular}

Jornadas de Formación e Innovación Docente del Profesorado | № 2 (2019) Esta obra se distribuye con la licencia Creative Commons Reconocimiento-NoComercial-SinObraDerivada Internacional (CC BY-NC-ND 4.0.) 


\begin{tabular}{|c|c|c|c|c|c|c|}
\hline 20 & +1 & & +2 & -1 & & \\
\hline 21 & +1 & +1 & & +2 & +1 & +1 \\
\hline 22 & & & & & +2 & +1 \\
\hline 23 & & -1 & +1 & +2 & +2 & \\
\hline 24 & +1 & & +1 & +1 & & +1 \\
\hline 25 & & +1 & +1 & +2 & +2 & +1 \\
\hline 26 & & & +2 & +1 & & +1 \\
\hline 27 & +2 & & +2 & +1 & +2 & +1 \\
\hline 28 & +1 & & +2 & +2 & & \\
\hline
\end{tabular}

De ambas tablas y de los gráficos de las escaleras, fácilmente se puede deducir que la pregunta no2 es la que presenta mayores dificultades y la 5 o en la que se produce un mayor avance. También existe la posibilidad de evaluar de un modo bastante objetivo el progreso total en el proceso usando los porcentajes, como en la Tabla 4, donde se usan colores para matizar los aspectos positivos (en verde) y negativo (en amarillo y naranja) de cada pregunta.

Tabla 4. Progreso general

\begin{tabular}{|c|c|c|c|c|c|c|c|}
\hline Preguntas & 1 & 2 & 3 & 4 & 5 & 6 & Media \\
\hline No subió de escalón & $40 \% *$ & $62 \% *$ & $41 \%$ & $30 \% *$ & $22 \%$ & $43 \% *$ & $39 \%$ \\
\hline Subió 1 escalón & $47 \%$ & $32 \%$ & $28 \%$ & $43 \%$ & $20 \%$ & $53 \%$ & $38 \%$ \\
\hline Subió 2 escalones & $13 \%$ & $3 \%$ & $24 \%$ & $27 \%$ & $55 \%$ & $0 \%$ & $20 \%$ \\
\hline Subió 3 escalones & $0 \%$ & $3 \%$ & $7 \%$ & $0 \%$ & $3 \%$ & $4 \%$ & $3 \%$ \\
\hline Aumento del área & $22 \%$ & $12 \%$ & $21 \%$ & $19 \%$ & $26 \%$ & $25 \%$ & $21 \%$ \\
\hline
\end{tabular}

* En estos casos, hay un alumno que baja un escalón

Jornadas de Formación e Innovación Docente del Profesorado | № 2 (2019) Esta obra se distribuye con la licencia Creative Commons Reconocimiento-NoComercial-SinObraDerivada Internacional (CC BY-NC-ND 4.0.) 


\section{Evaluación del CMD}

La experiencia ha sido claramente positiva, prueba de ello es que si tuviese que volver a hacer-mejor dicho, cuando vuelva a hacer- el mapa de contenidos, el modelo metodológico y el cuestionario de evaluación, serán distintos de los que he expuesto en este artículo. En otras palabras, el curso es intrigante y despierta inquietudes docentes.

Las reflexiones sobre los componentes del aprendizaje y el comprender cómo es este proceso han abierto un mundo de posibilidades que poco a poco espero ir experimentando e incorporando en mi docencia: la importancia del componente actitudinal, el hacer que los alumnos sean partícipes de su propio aprendizaje, o el proporcionarles los instrumentos para que ellos avancen (o al menos lo intenten) por sí solos, han demostrado ser procedimientos docentes de una enorme potencia.

Aunque intuitivamente muchas de las técnicas que he aprendido ya las estaba aplicando en mayor o menor medida, carecía del conocimiento preciso para comprenderlas, desarrollarlas y extrapolarlas correctamente a otras asignaturas.

Respecto a años anteriores, los cambios que he realizado en el CMD los podemos resumir en las siguientes: 1) abrir las sesiones con preguntas clave para captar la atención y fomentar la participación de los alumnos; 2) iniciar las prácticas lo más pronto posible incorporando las explicaciones teóricas en las menores y más numerosas dosis que iban requiriendo durante su desarrollo; y 4) dejar el resto de las exposiciones teóricas para el final. El primer cambio mejora el ambiente e implica más al alumno mejorando su actitud pero, a cambio requiere una mayor cantidad de tiempo que no tenemos. Adelantar la práctica y combinarla con el contenido teórico ha dado unos frutos 
muy positivos y los alumnos se han implicado en el aprendizaje. Por último, retrasar la teoría ha tenido unos resultados peores de lo esperado, seguramente debido a las prisas para intentar compensar el tiempo (con sus correspondientes los contenidos) perdidos en las sesiones anteriores. Por ello ha quedado como un "cajón de sastre" donde se ha perdido la atención y con ella el espíritu participativo del alumno.

En el futuro pretendo incorporar pequeños cambios en esta y en las otras asignaturas que imparto:

- Proporcionar índice de contenidos por sesiones para estructurar y proporcionar a los alumnos un guion con su contenido, actividades y tareas a realizar.

- Comenzar las clases con una pregunta o actividad que intente captar la atención del estudiante y le implique en el aprendizaje.

- Adelantar todo lo posible las prácticas intentando incorporar en ellas el contenido teórico preciso en fases sucesivas.

- Incorporar la tarea de la lectura de los temas antes de su exposición teórica.

- Reducir los contenidos teóricos y relacionarlos con las posibilidades que pudiesen ofrecer en una práctica distinta.

Apenas he hablado del sistema de calificación ya que en esta materia se basa directamente en la aplicación de los contenidos y se plantean cuestiones muy abiertas y creo que mejores que en el cuestionario expuesto. Pese a ello, creo que la experiencia con los sistemas de evaluación y especialmente con las escaleras ha sido muy enriquecedora. Son instrumentos muy potentes que espero poder desarrollar mejor para comprobar la validez de los diversos instrumentos docentes. 


\section{Referencias bibliográficas.}

Bain, K (2007). Lo que hacen los mejores profesores universitarios. Valencia: Publicacions de la Universitat de València.

Carr, N. G. (2011). Superficiales. ¿Qué está haciendo Internet con nuestras mentes? Madrid: Santillana Ediciones Generales, S.L.

De Alba, N. y Porlán, R. (2017). La metodología de enseñanza. En R. Porlán (Coord.). Enseñanza universitaria. Cómo mejorarla (pp. 37-53). Madrid: Morata.

Finkel, D. (2008). Dar clase con la boca cerrada. Valencia: Publicacions de la Universitat de València.

Rivero, A. y Porlán, R. (2017). La evaluación en la enseñanza universitaria. En R. Porlán (Coord.). Enseñanza universitaria. Cómo mejorarla (pp. 73-91). Madrid: Morata.

Jornadas de Formación e Innovación Docente del Profesorado | № 2 (2019) Esta obra se distribuye con la licencia Creative Commons Reconocimiento-NoComercial-SinObraDerivada 4.0 Internacional (CC BY-NC-ND 4.0.) 